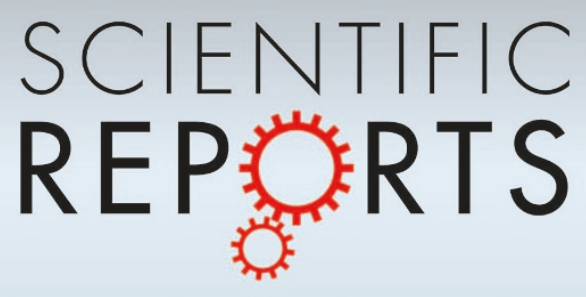

OPEN

SUBJECT AREAS:

CARDIOVASCULAR

MODELS

IMMUNOCHEMISTRY

BIOMATERIALS-CELLS

EXPERIMENTAL MODELS OF

DISEASE

Received

27 February 2013

Accepted

11 June 2013

Published

28 June 2013

Correspondence and requests for materials should be addressed to

J.L.M. (MehtalL@

UAMS.edu) or X.D. (dengxy1953@buaa. edu.cn)

\footnotetext{
* These authors contributed equally to
} this work.

\section{Concentration polarization of ox-LDL activates autophagy and apoptosis via regulating LOX-1 expression}

\author{
Zufeng Ding ${ }^{1,2 *}$, Shijie Liu'*, Changqing Sun ${ }^{3}$, Zengsheng Chen², Yubo Fan ${ }^{2}$, Xiaoyan Deng ${ }^{2}$, \\ Xianwei Wang' \& Jawahar L. Mehta ${ }^{1}$
}

${ }^{1}$ Central Arkansas Veterans Healthcare System and the Department of Medicine, University of Arkansas for Medical Sciences, Little Rock, Arkansas, ${ }^{2}$ Key Laboratory for Biomechanics and Mechanobiology of Ministry of Education, School of Biological Science and Medical Engineering, Beihang University, Beijing 100191, PR China, ${ }^{3}$ College of Public Health, Zhengzhou University, 100 Kexue Avenue, Zhengzhou 450001, Henan, PR China.

Here we demonstrate that "concentration polarization" of ox-LDL enhances LOX-1 expression and ox-LDL uptake. It damages cell surface heparan sulfate proteoglycans (HSPG) and activates LOX-1 dependent autophagy and apoptosis. We also show that ox-LDL concentration polarization occurs on the surface of rabbit thoracic aorta and induces autophagy and apoptosis. In order to investigate the significance of swirling flow on LOX-1 expression, HSPG damage, autophagy and apoptosis in the arterial system, an ex vivo model of swirling flow was developed. We observed that swirling flow decreases relative wall concentration of ox-LDL, inhibits LOX-1 expression, protects HSPG from damage, and decreases both autophagy and apoptosis. Taken together, our data suggest that ox-LDL concentration polarization plays an important role in the localization of atherosclerotic lesions concomitant with LOX-1 dependent autophagy and apoptosis. These observations also suggest a novel mechanism by which swirling flow in the arterial system protects arterial wall from atherogenesis.

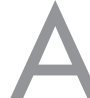

therosclerotic lesions are known to occur in a spatially heterogeneous fashion, developing preferentially at the inner wall of curved segments and outer walls of bifurcations ${ }^{1}$. It has been proposed that shear stress dependent modulation of atherogenesis relies on the formation of a low density lipoprotein (LDL)-rich layer, a result of so called 'concentration polarization', which is adjacent to the luminal surface of the arterial channel $^{2,3}$. The formation of such a layer is considered possible because there is a two orders of magnitude imbalance between the measured velocity of water filtration $\left(\sim 4 \times 10^{-6} \mathrm{~cm} / \mathrm{s}\right)^{4}$ and the permeability of LDL across the endothelium $\left(\sim 2 \times 10^{-8} \mathrm{~cm} / \mathrm{s}\right)^{5}$. Deng et $\mathrm{al}^{6}$ predicted this mass transport phenomenon of concentration polarization of LDL and suggested that it plays an important role in the localization of atherosclerotic lesions.

As the major receptor responsible for binding, internalizing and degrading oxidatively modified low density lipoprotein (ox-LDL), lectin-like ox-LDL scavenger receptor-1 (LOX-1) plays a key role in the genesis and development of atherosclerosis ${ }^{7,8}$. Activation of LOX-1 has been implicated in vascular cell proliferation, alteration in cell cycle signals and apoptosis ${ }^{9,10}$.

Autophagy is an evolutionary conserved process involved in the degradation of excess or dysfunctional organelles ${ }^{11,12}$. Under normal conditions in most vascular cells, autophagy is an important house-keeping process and may be considered a cell survival program ${ }^{11,12}$. Although excessive autophagic activity leads to total collapse of all cellular functions and induction of autophagic death, moderately enhanced autophagy can promotes cell survival $^{11-13}$. Apoptosis, as a process of programmed cell death, is meant to remove cells exposed to noxious stimuli, such as ox-LDL, and thus plays an important role in many pathological conditions including atherosclerosis $^{9,14,15}$. The regulation of autophagy and apoptosis in response to ox-LDL might be of interest in understanding endothelial cell biology in atherosclerotic regions wherein the concentrations of ox-LDL are high ${ }^{9,10}$.

Endothelial luminal surface, which is constantly exposed to the mechanical forces generated by blood flow, plays important role in the regulation of vascular tone and blood vessel remodeling, binding of lipoproteins and activation of inflammatory responses; all these processes are involved in atherogenesis ${ }^{16-18}$. Previously, we showed that besides LDL concentration polarization, a similar phenomenon of ox-LDL concentration polarization exists on the luminal surface of smooth muscle cells ${ }^{19}$. However, to the best of our knowledge, there is no report on the 
effect of ox-LDL concentration polarization on LOX-1 mediated autophagy and apoptosis either in isolated cells or in animal models. Further, there are no data on the relationship between shear stress and LOX-1 expression in response to ox-LDL on the luminal surface of cultured endothelial cells.

We hypothesized that concentration polarization of ox-LDL could activate LOX-1 mediated autophagy and apoptosis, which may be an initial step of atherogenesis. The present study was, therefore, designed to investigate this hypothesis. In addition, we examined the impact of swirling blood flow on wall shear stress and cell surface concentration polarization of ox-LDL.

\section{Results}

Water filtration rate $\left(v_{w}\right)$ and wall concentration of DiI-ox-LDL $\left(c_{w}\right)$. In order to verify that concentration polarization of ox-LDL occurs at the surface of HUVECs, water filtration rate $\left(v_{w}\right)$ and wall concentration of DiI-ox-LDL $\left(c_{w}\right)$ were measured. Figure $1 \mathrm{~B}$ shows the $v_{w}$ across the cell monolayer for the permeable group with DiIox-LDL ranging from 0 to $60 \mu \mathrm{g} / \mathrm{ml}$. As shown, the average $v_{w}$ was about $23.3 \times 10^{-6} \mathrm{~cm} / \mathrm{s}$. For the non-permeable group, the water filtration rate was zero (data not shown). Figure 1C shows wall concentration of DiI-ox-LDL on the surface of HUVECs in the permeable group. The relative wall concentration for the permeable group, $c_{w} / c_{0}$, was higher than 1.0 , indicating that concentration polarization of DiI-ox-LDL occurred on the surface of cells. Due to lack of filtration flow across the HUVECs layer, $c_{w} c_{0}$ was always 1.0 for the non-permeable group (data not shown).

Ox-LDL concentration polarization, LOX-1 expression and ox-LDL uptake. As shown in Figure 1D, ox-LDL induced a concentration-dependent increase in LOX-1 expression. Due to concentration polarization of ox-LDL on the cell surface, LOX-1 expression was higher $(\approx 30 \%)$ in the permeable group compared with the non-permeable group at all concentrations. Of note, ox-LDL uptake in HUVECs correlated positively with the concentration of ox-LDL for both groups, but ox-LDL uptake in the permeable group was greater (by $\approx 33 \%$ ) than in the non-permeable group (Figure 1E).

Concentration polarization of ox-LDL, heparan sulfate proteoglycans and autophagy. As important components of the cell surface, extracellular matrix and basement membrane, heparan sulfate proteoglycans (HSPG) regulate a wide variety biological activities, including basement membrane permeability; cell migration, adhesion and proliferation; receptor interaction; and lipoprotein

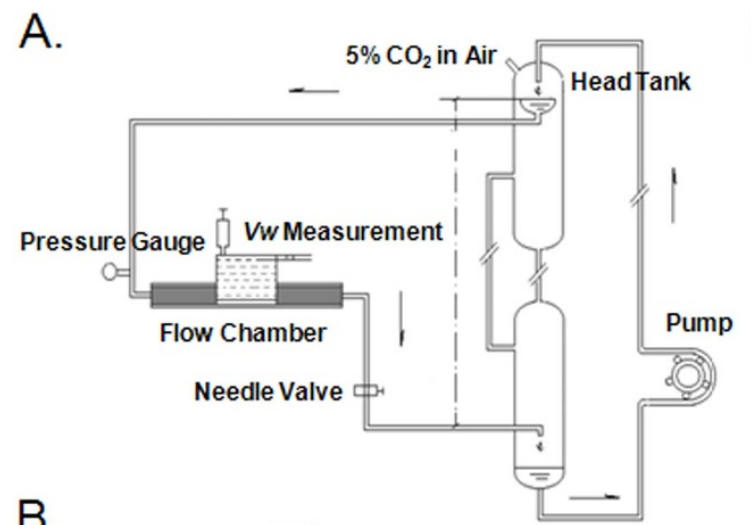

D.

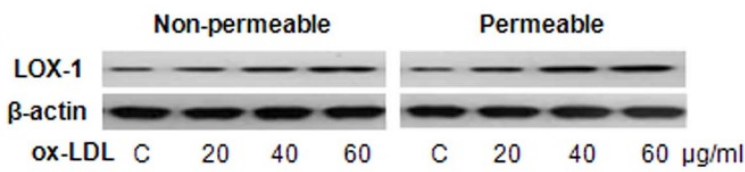

B.
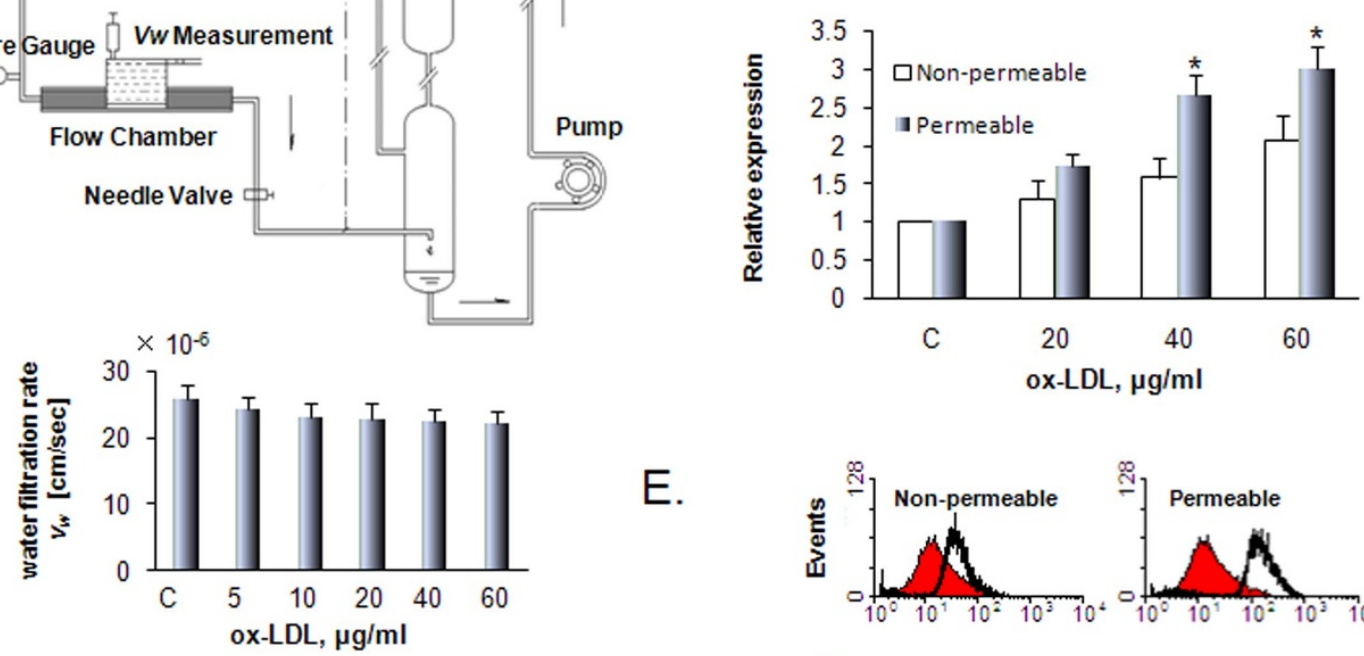

E.

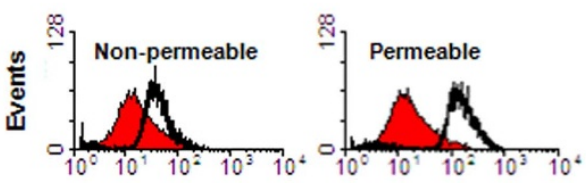

C.
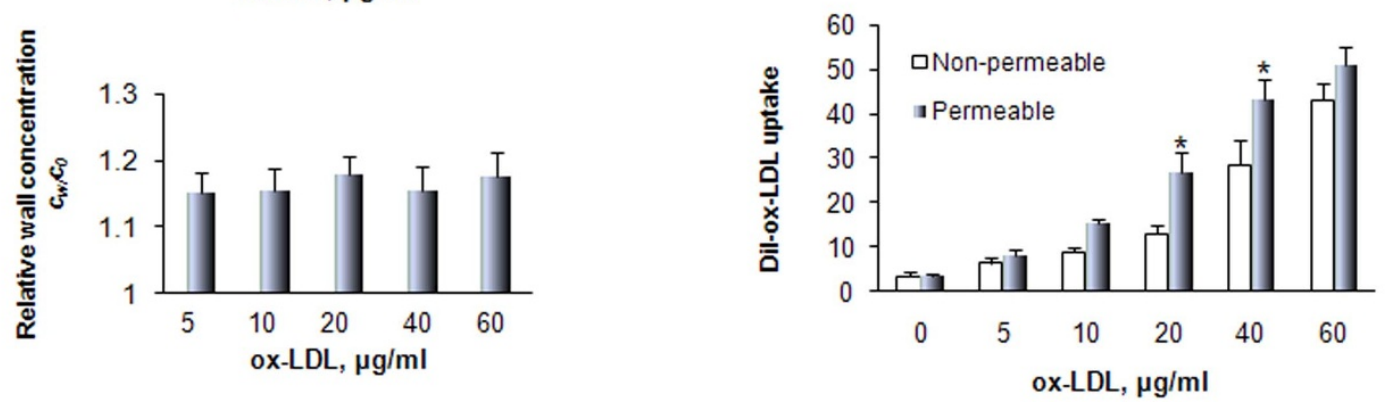

Figure 1 Concentration polarization of ox-LDL increases LOX-1 expression and ox-LDL uptake. (A) Schematic drawing of the experimental perfusion system. The overflow head-tank provides a steady flow to HUVECs and thoracic aorta segment. (B) Water filtration rate ( $\left.v_{w}\right)$ and $(\mathrm{C})$ relative wall concentration $\left(c_{w} / c_{0}\right)$ of HUVECs in permeable group. (D) and (E) Note a significantly greater increase in LOX-1 expression and DiI-ox-LDL uptake in the permeable group compared with the non-permeable group. The samples were derived from the same experiment and gels/blots were processed in parallel. HUVECs were treated with increasing concentration of ox-LDL for 24 hours under steady shear stress and constant perfusion pressure within the flow chamber kept at $1.3 \mathrm{~Pa}$ and $100 \mathrm{mmHg}$, respectively. Bar graphs represent data in mean $\pm \mathrm{SD}$ based on 5 experiments, ${ }^{*} \mathrm{P}<0.05 \mathrm{vs}$. non-permeable group. 
binding and uptake ${ }^{20}$. We hypothesized that concentration polarization of ox-LDL may damage and decrease cell surface HSPG leading to an increase in membrane permeability with seepage of ox-LDL into the arterial wall. To verify this hypothesis, HSPG specific antibody was used to stain cell surface HSPG and measure its expression in both non-permeable and permeable groups of cells. As shown in Figure 2A, ox-LDL damaged (and decreased) HSPG in a dose-dependent manner. The decrease in the permeable group was greater (by $\approx 36 \%$ ) than in the nonpermeable group. Combined with the aforementioned results, it can be concluded that HSPG damage induces ox-LDL accumulation within the HUVECs layer, and then enhances LOX-1 expression (Figure 1D), and ox-LDL uptake (Figure 1E).

Next, we assessed autophagy in response to concentration polarization of ox-LDL by measuring three recognized autophagosome markers, LC3, beclin-1, and Atg $5^{14-16}$. As shown in Figure 2B-D, oxLDL induced LC3-II, beclin-1 and Atg5 expression in a concentration-dependent manner. Further, ox-LDL induced expression of these autophagy makers in the permeable group was greater (by $\approx 25-60 \%$ ) than in the non-permeable group.
Concentration polarization of ox-LDL and apoptosis. Previous studies have shown that ox-LDL induces apoptosis in endothelial cells via LOX-1 activation ${ }^{12}$. Apoptosis is regulated by various proapoptosis proteins, such as Cytochrome c, Caspase-3 and Bax, and anti-apoptosis proteins, such as $\mathrm{Bcl}-2$ and $\mathrm{Bcl}-\mathrm{xL}^{21}$. In our study, we observed that ox-LDL in a concentration-dependent manner inhibited the expression of $\mathrm{Bcl}-2$ and $\mathrm{Bcl}-\mathrm{xL}$ (Figure 3A and $\mathrm{B}$ ) and induced the expression of $\mathrm{Bax}$ and Caspases in both two groups (Figure 3C and D). Further, ox-LDL inhibited Bcl-2 and $\mathrm{Bcl}$-xL more (by $\approx 20-35 \%$ ) in the permeable group than in the non-permeable group. Ox-LDL increased Bax and Caspases, again more (by $\approx 20-25 \%$ ) in the permeable group than in the nonpermeable group (Figure 3A-D).

Next, we studied the development of autophagy and apoptosis in relation to the concentration of ox-LDL in parallel experiments in the 2 groups. We observed that the number of cells expressing apoptosis increased sharply in response to 10 to $60 \mu \mathrm{g} / \mathrm{ml}$ of ox-LDL, and reached a plateau upon exposure to $>60 \mu \mathrm{g} / \mathrm{ml}$ concentration of ox-LDL (Figure 3E). On the other hand, the number of endothelial cells showing autophagy increased in response to 20 to $60 \mu \mathrm{g} / \mathrm{ml}$ of
A.

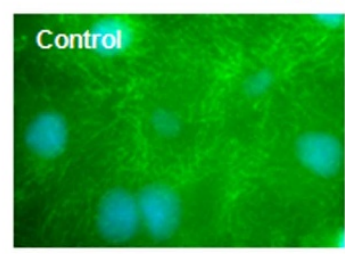

Heparan Sulfate Proteoglycans (HSPG)
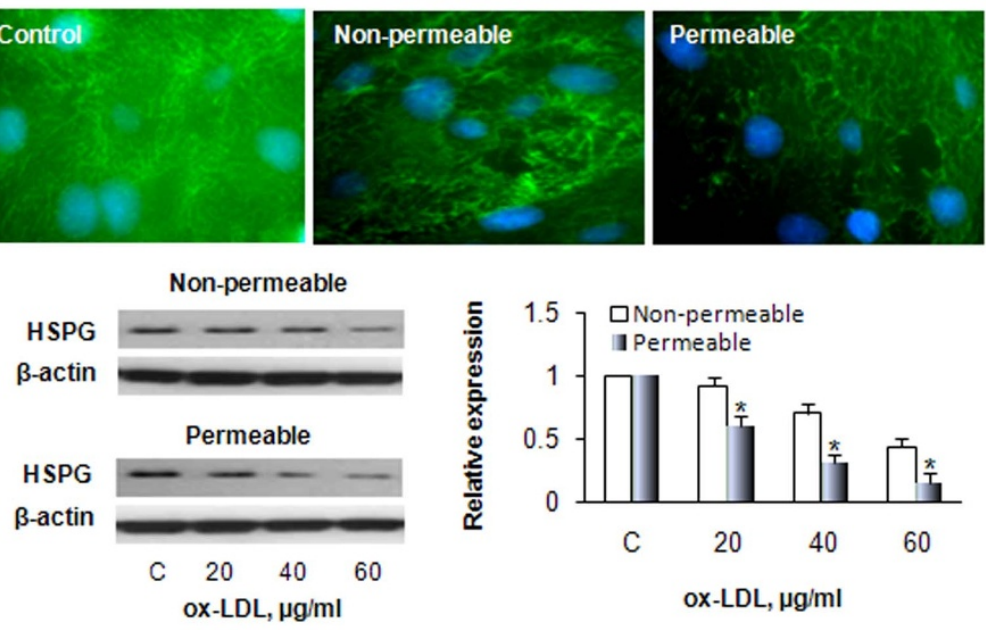

B.

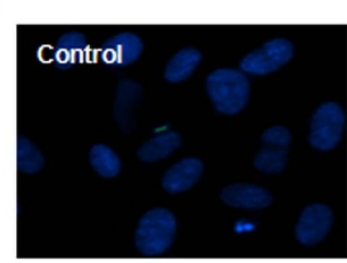

LC3B expression
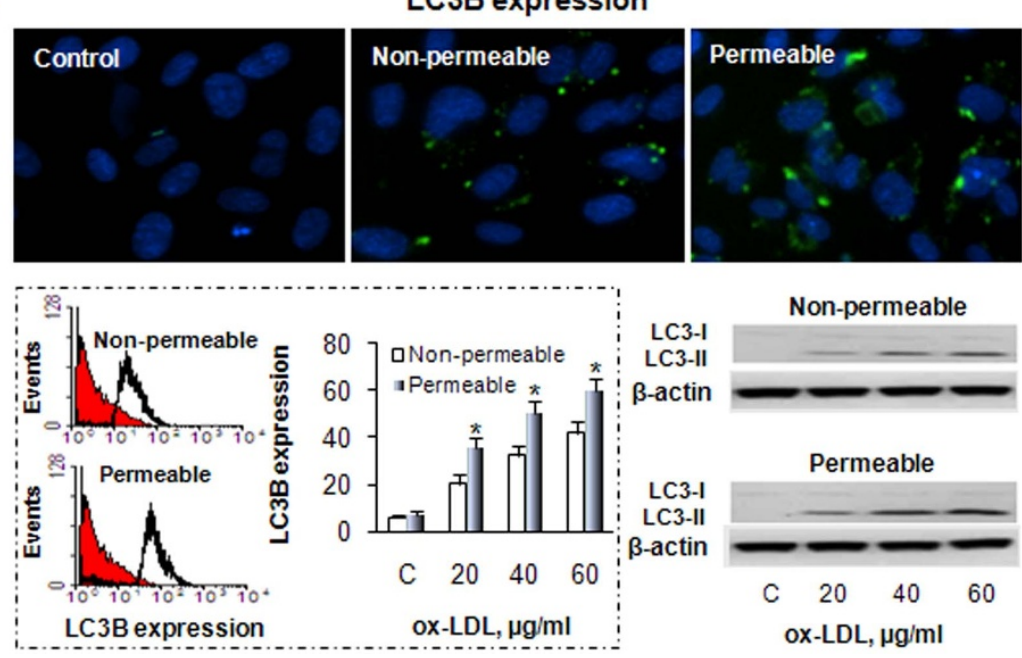

C.

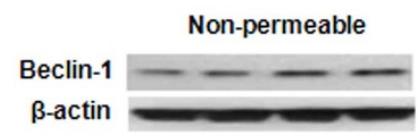

Permeable
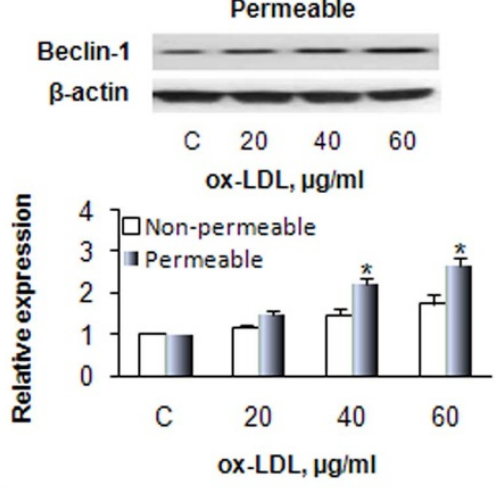

D.
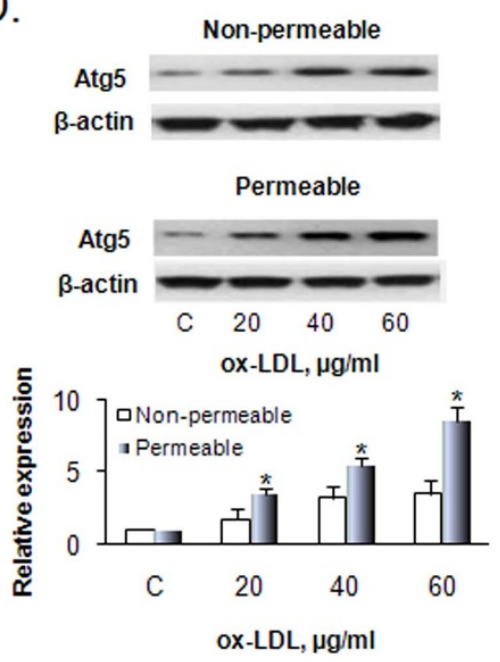

Figure $2 \mid$ Concentration polarization of ox-LDL induces autophagy. (A) Cell surface HSPGs were extensively damaged and their expression decreased sharply, significantly more in permeable group compared with non-permeable group. Hoechst 33342 was used to stain cell nuclei. (B-D) HUVECs show greater expression of LC3-II/LC3-I, Beclin-1 and Atg5, in the permeable group compared with the non-permeable group. The samples were derived from the same experiment and that gels/blots were processed in parallel. Bar graphs represent data in mean \pm SD based on 5 experiments, ${ }^{*} \mathrm{P}<0.05 \mathrm{vs}$. non-permeable group. 
A.

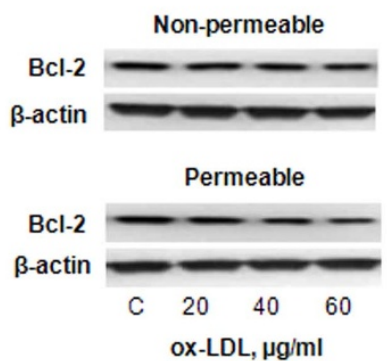

B.

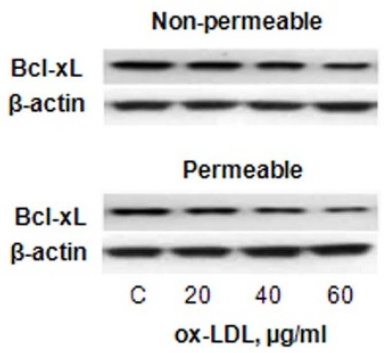

C.
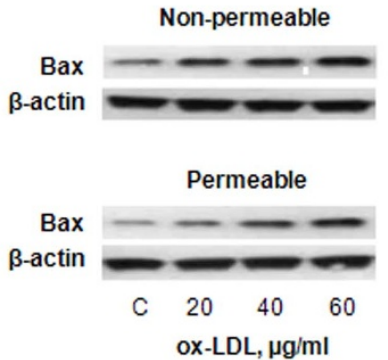
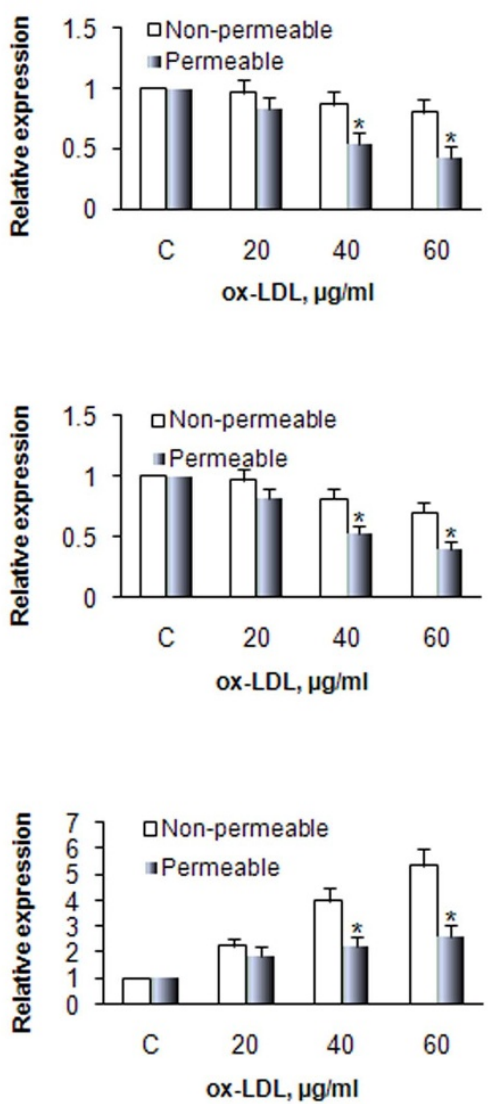

E.

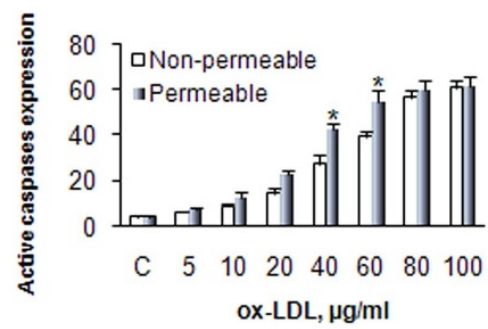

F.

D.
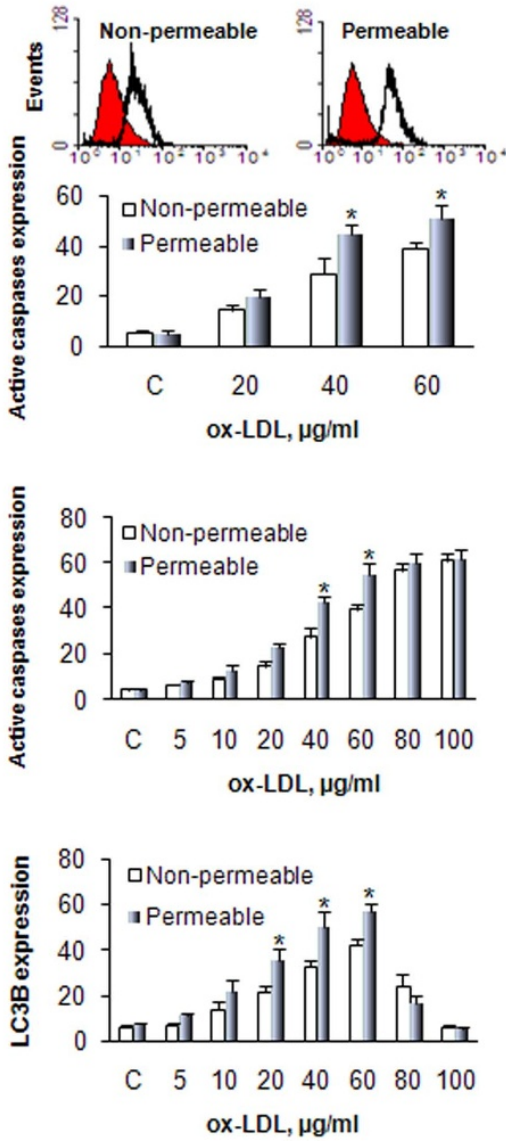

Figure 3 Concentration polarization of ox-LDL induces apoptosis. (A) and (B) Ox-LDL decreases expression of anti-apoptotic proteins Bcl-2 and Bcl$\mathrm{xL}$, significantly more in the permeable group than in the non-permeable group. (C) and (D) Ox-LDL increases expression of pro-apoptotic proteins Bax and Caspases, significantly more in the permeable group than in the non-permeable group. The samples derive from the same experiment and gels/blots were processed in parallel. (E) and (F) Patterns of ox-LDL-mediated apoptosis and autophagy. In the non-permeable group, ox-LDL increases apoptosis rate in a dose-dependent manner up to $60 \mu \mathrm{g} / \mathrm{ml}$, and then the apoptosis rate stabilizes. Ox-LDL $(5-60 \mu \mathrm{g} / \mathrm{ml})$ increases autophagy, but at higher concentrations autophagy sharply falls. Due to the occurrence of concentration polarization of ox-LDL in permeable group, the changes in apoptosis markers are more pronounced than in the non-permeable group. Bar graphs represent data in mean $\pm \mathrm{SD}$ based on 5 experiments, $* \mathrm{P}<0.05$ vs. nonpermeable group.

ox-LDL, and then declined rapidly as the concentration of ox-LDL was increased further. It is of note that apoptosis and autophagy were always greater in the permeable group compared with the non-permeable group.

Apoptosis and autophagy in rabbit thoracic aorta. To investigate whether concentration polarization of ox-LDL occurs at the endothelial surface, the straight segment of rabbit thoracic aorta was perfused as described in Figure 1A. First, water filtration rate $\left(v_{w}\right)$ and relative wall concentration of ox-LDL $\left(c_{w a} / c_{0}\right)$ were measured as described previously ${ }^{6} . C_{0}$ is the bulk concentration of ox-LDL in the perfusion solution. As shown in Figure 4A and 4B, the average $v_{w}$ and $c_{w a} / c_{0}$ were $12.9 \times 10^{-6} \mathrm{~cm} / \mathrm{s}$ and 1.19 , respectively, indicating that concentration polarization occurred on the luminal surface of aorta.

Subsequently, expression of HSPG, LOX-1, autophagy- and apoptosis-related proteins were measured. As shown in Figure 4C, concentration polarization of ox-LDL decreased HSPG expression in a concentration-dependent fashion. Similarly to the cultured cell experiments, concentration polarization of ox-LDL via LOX-1 activated autophagy (expression of LC3, Beclin-1 and Atg5) and apoptosis (expression of Cytochrome c, Bax, Bcl-2 and Bcl-xL).

Swirling flow and ox-LDL concentration polarization. Since flow patterns impact the development of atherosclerosis, and concentration polarization of ox-LDL can aggravate LOX-1 expression within arteries and initiate autophagy and apoptosis, we used a model of swirling flow to investigate the effect of swirling flow on LOX-1 mediated autophagy and apoptosis (Figure 5A).

Helicity. In order to better characterize the swirling flow model, helicity calculated by the following equation was used to assess swirling flow ${ }^{22}$.

$$
H=(\nabla \times \vec{V}) \cdot \vec{V}
$$

The area-weighted average helicity of the flow at different crosssections along the vessel is shown in Figure 5B. The helicity of the swirling flow created by the flow guider was highest at the origin of the test vessel, decreased along the vessel and dropped drastically after $10 \mathrm{~mm}$.

General flow patterns. Next, flow patterns (velocity vectors at $5 \mathrm{sec}-$ tions along the vessels) were measured for the swirling flow and normal flow groups. As shown in Figure 5C, there was swirling flow pattern in the swirling flow group, but not in the control (normal flow) group. Compared with the normal flow, swirling flow was associated with altered velocity profiles in the aortic segment and the maximum velocity profile shifted away from the center of the aortic segment. This simulation also revealed that the flow created by 
A.

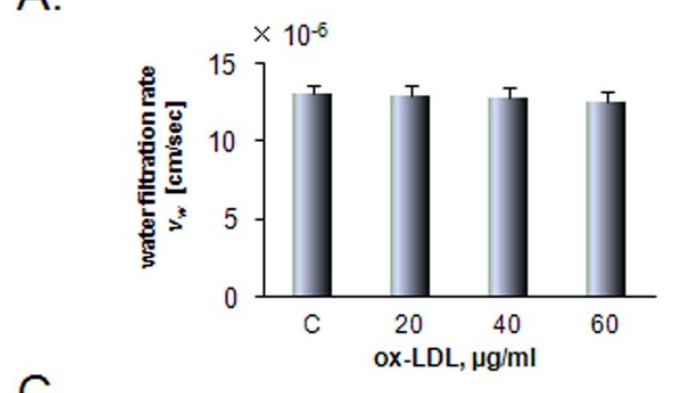

B.

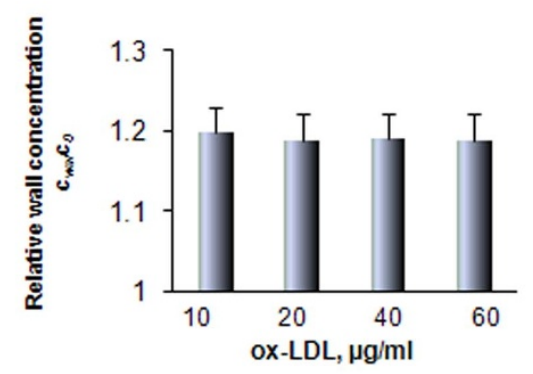

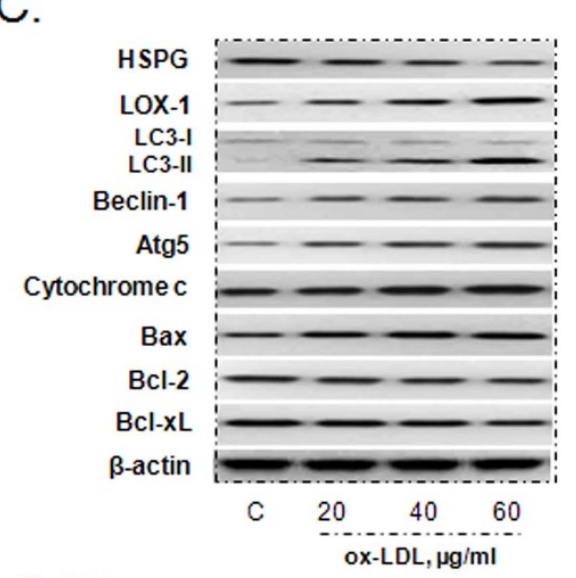
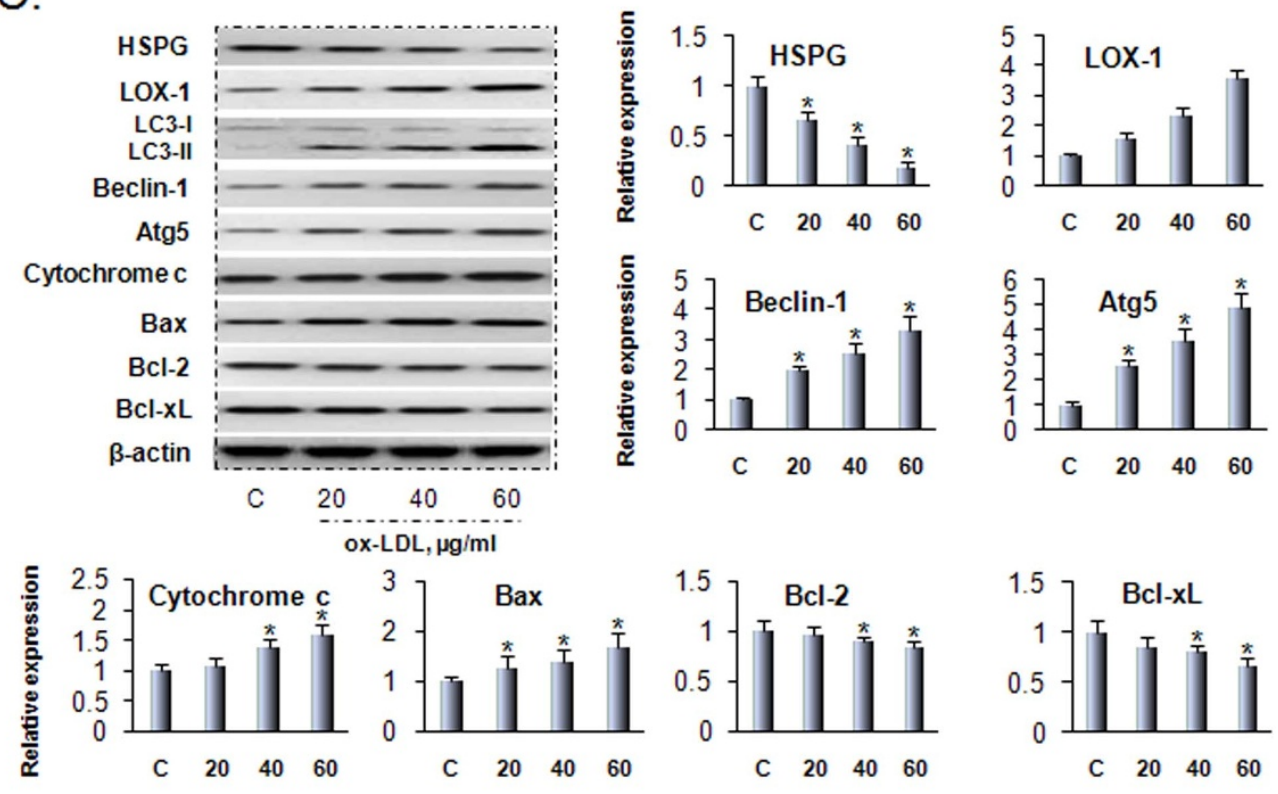

ox-LDL, $\mu \mathrm{g} / \mathrm{ml}$

Figure $4 \mid$ Concentration polarization of ox-LDL occurs on the surface rabbit thoracic aorta. (A) and (B) show water filtration rate ( $v_{w}$, about $\left.12.9 \times 10^{-6} \mathrm{~cm} / \mathrm{s}\right)$ and relative ox-LDL wall concentration $\left(c_{w a} / c_{0}\right.$, always higher than 1.0), indicating that concentration polarization of ox-LDL also occurred on the surface rabbit thoracic aorta. (C) Concentration polarization of ox-LDL decreases HSPG expression, and increases LOX-1 expression and induces activation of autophagy (expression of LC3, Beclin-1 and Atg5) and apoptosis (expression of Cytochrome c, Bax, Bcl-2 and Bcl-xL). The samples were derived from the same experiment and gels/blots were processed in parallel. Bar graphs represent data in mean \pm SD based on 7 experiments, $* \mathrm{P}<0.05$ vs. control.

the swirling flow guider was progressively less along the aortic segment.

Relative wall concentration $\left(V_{w}\right.$ and $\left.c_{w}\right)$. Though $v_{w}$ was similar in both normal flow and swirling flow groups $\left(13.4 \times 10^{-6} \mathrm{~cm} / \mathrm{s}\right.$ and $12.5 \times 10^{-6} \mathrm{~cm} / \mathrm{s}$, respectively (Figure 5D), the relative wall concentration $\left(c_{w a} / c_{0}\right)$ in swirling flow group was lower by about $50 \%$ compared with normal flow group (Figure 5E).

Swirling flow and autophagy and apoptosis. As shown in Figure 5F, swirling flow protected HSPG from damage induced by ox-LDL compared with normal flow group. Swirling flow also inhibited oxLDL mediated increase in LOX-1 expression, and expression of autophagy (LC3, Beclin-1 and Atg5) and apoptosis (Cytochrome c, Bax, Bcl-2 and Bcl-xL).

Concentration polarization of ox-LDL and LOX-1 related upstream signals. An increasing body of evidence indicates that ROS are key signaling molecules in LOX-1-mediated signaling pathways, and NADPH oxidase (such as $\mathrm{p} 22^{\text {phox }}$ and $\mathrm{p} 47^{\text {phox }}$ subunits) activation is a major source of ROS in endothelial cells ${ }^{10}$. Therefore, we investigated the relationship between concentration polarization of ox-LDL and intracellular ROS generation as well as
NADPH oxidase expression. Results from flow cytometry and western blot showed that ox-LDL induced a marked increase in ROS generation (Figure 6A) as well as the expression of NADPH oxidase subtypes p22 $2^{\text {phox }}$ (Figure 6B) and p47 $7^{\text {phox }}$ (Figure 6C), all more (by $\approx 30-40 \%$ ) in the permeable group than in the nonpermeable group.

Ox-LDL binding to LOX-1 activates mitogen activated protein kinases (MAPKs) and nuclear factor-kappaB (NF- $\kappa \mathrm{B})$, which in turn induces further LOX-1 expression and enhances its activity ${ }^{10}$. In the present study, we found that, ox-LDL induced a marked concentration-dependent increase in phosphorylated p38 MAPK (Figure 6D) and phosphorylated NF- $\mathrm{B}$ (Figure $6 \mathrm{E}$ ), both more (by $\approx 30-45 \%$ ) in the permeable group than in the non-permeable group.

\section{Discussion}

It has been suggested that atherosclerotic lesions develop preferentially at curved and bifurcations segments ${ }^{1}$, and flow-induced shear stress is one of the most important hemodynamic factors in the localization of atherogenesis ${ }^{23}$. To explain the phenomenon of localization of atherogenesis, Deng et $\mathrm{al}^{6}$ first predicted and then demonstrated a mass transport phenomenon of 'concentration polarization' of atherogenic LDLs with the highest LDL concentration in 
A.

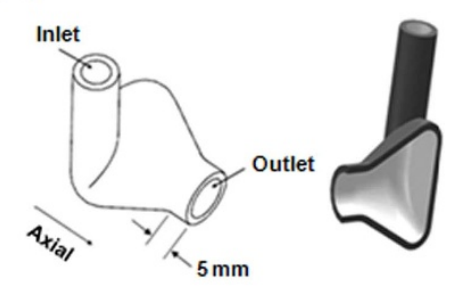

D.

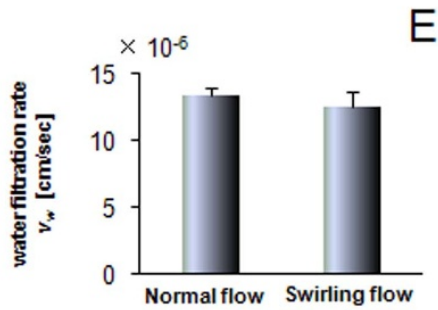

F.

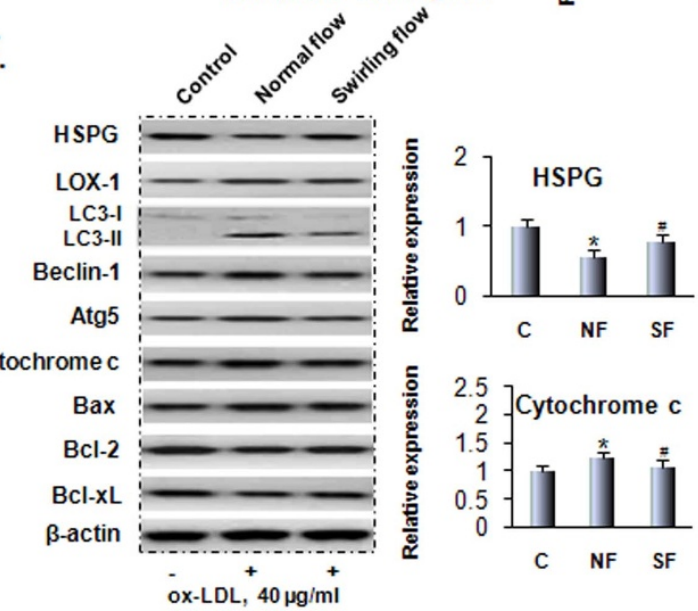

B.

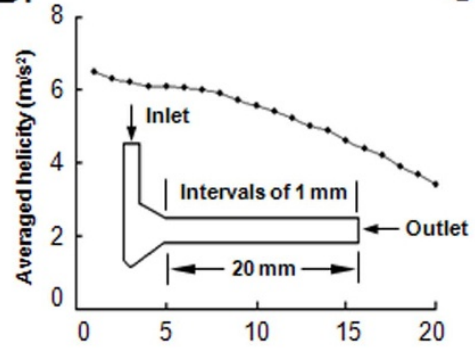

E.

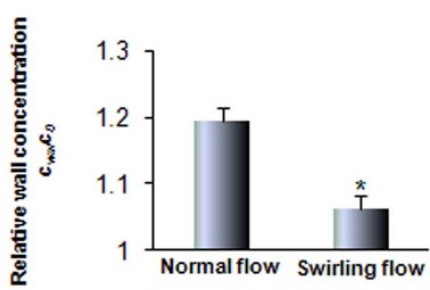

C.

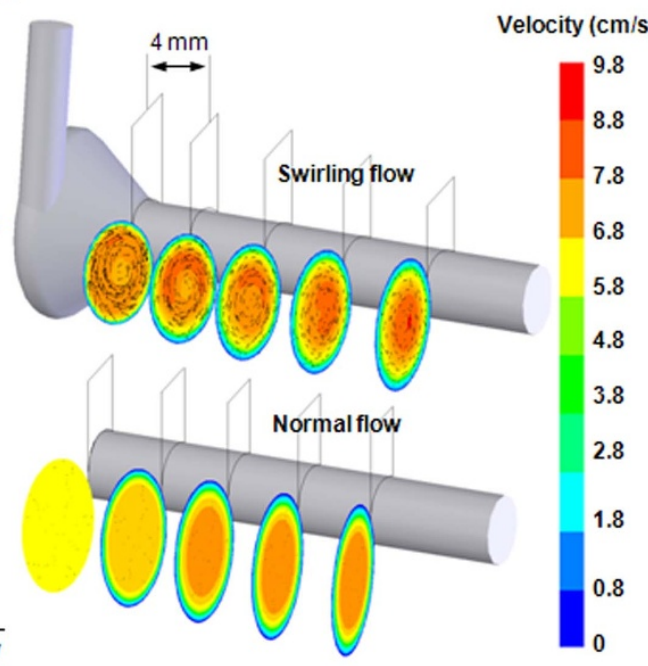

Figure 5 Swirling flow inhibits autophagy and apoptosis via decreasing wall concentration of ox-LDL on the surface of rabbit thoracic aorta. (A) The model of the spiral flow guider. The geometrical parameters of the model were based on the measurements of the rabbit thoracic aorta. (B) Plot of areaweighted average of helicity from the origin of the spiral flow model along the aortic segment. The origin is shown as 0 along the $20 \mathrm{~mm}$ length of aortic segment. (C) Velocity vector features at 5 cross-sections perpendicular to the tube center axis in the 2 groups, colored by velocity magnitude, showing an apparent helical flow in the aortic segment with the flow guider. Flow velocity in the aortic segment has been redistributed due to the rotation of the flow. (D) Water filtration rate in the two groups. (E) Swirling flow obviously decreases wall concentration of ox-LDL compared with normal flow. (F) Swirling flow protects HSPG damage from ox-LDL, decreases LOX-1 expression, regulates autophagy (expression of LC3, Beclin-1 and Atg5) and apoptosis (expression of Cytochrome c, Bax, Bcl-2 and Bcl-xL). The samples were derived from the same experiment and gels/blots were processed in parallel. Abbreviations: C, NF and SF are control, normal flow and swirling flow, respectively. Bar graphs represent data in mean \pm SD based on 7 experiments, ${ }^{*} \mathrm{P}<0.05$ vs. control. ${ }^{\#} \mathrm{P}<0.05$ vs. normal flow.

the luminal surface (endothelium and sub-endothelial layers) which gradually decreased towards the adventitia.

LOX-1 mediated ox-LDL endocytosis is considered to play an essential role in the pathogenesis of atherosclerosis ${ }^{7-10}$. However, there is paucity of information on the regulation of LOX-1 expression and concentration polarization of ox-LDL. In this study, we investigated the relationship between concentration polarization of ox-LDL and LOX-1 expression and development of autophagy and apoptosis. We also examined the relationship of concentration polarization of ox-LDL and HSPG.

Our first observation was that concentration polarization of oxLDL enhanced LOX-1 expression, leading to ox-LDL uptake. Cell surface HSPG is known to regulate endothelial cell adhesion, migration and receptor interaction ${ }^{20}$. We showed that the concentration polarization of ox-LDL reduced HSPG and altered cell morphology in the HUVECs monolayer, more in the permeable group than in the non-permeable group. It is reasonable to assume that damage to HSPG and its decrease would result a prolonged interaction between
ox-LDL and cell surface receptors resulting in greater LOX-1 expression. Based on the HSPG degradation data in HUVECs monolayer, we found that LOX-1 expression was greater in the permeable group than in the non-permeable group of cells.

Autophagy plays an essential role in cell survival (or death) ${ }^{11-13}$, while apoptosis represents programmed cell death and is a major occurrence in various stage of atherosclerosis ${ }^{12,14,24}$. Although the precise role of autophagy and apoptosis in atherosclerosis is not known, both are often seen concomitantly in cells exposed to oxLDL as well as in atherosclerotic lesions ${ }^{9,10}$. We assessed autophagy by measuring three different markers, LC3, beclin-1, and Atg5, all found in endothelial cells and SMCs ${ }^{11}$. We observed that extensive expression of autophagy markers in HUVECs treated with ox-LDL, more in the permeable group than in the non-permeable group. Based on the observations presented here, we believe that autophagy in endothelial cells with concentration polarization of ox-LDL represents a stress adaptation that prevents cell death and serves to eliminate superfluous, damaged cells and organelles that would include 
A.

ROS generation: DHE staining
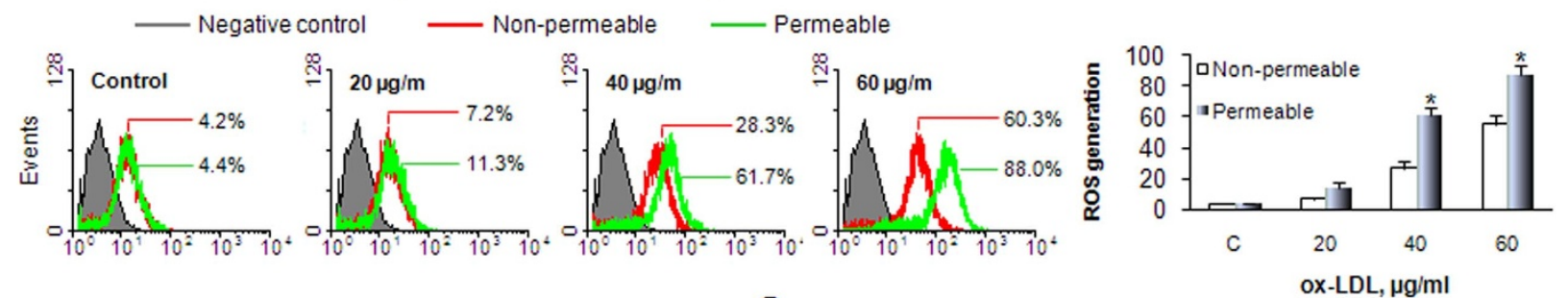

B.
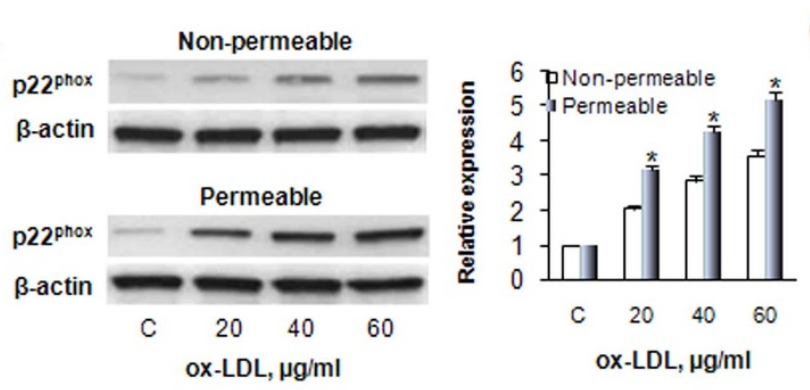

C.
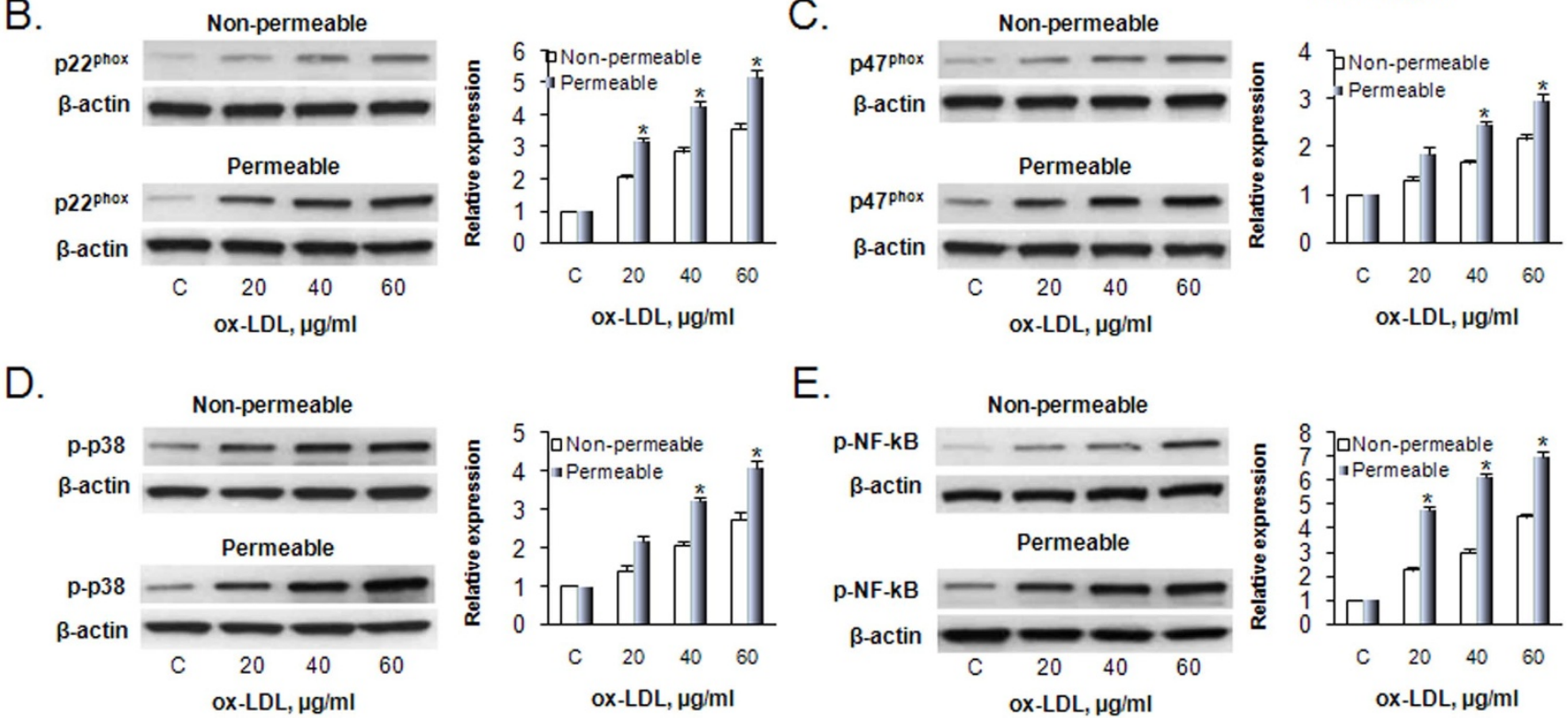

E.
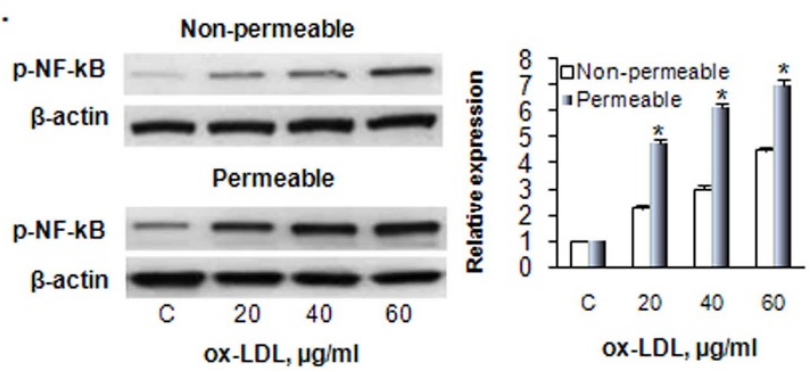

Figure 6 Concentration polarization of ox-LDL induces ROS generation as well as expression of NADPH oxidase subunits p22 ${ }^{\text {phox }}$ and p47 ${ }^{\text {phox }}$, p-p38 and p-NF-кB. (A) There is greater increase of ROS generation in the permeable than in the non-permeable group measured by flow cytometry. (B) to (E) Increased expression of NADPH oxidase subunits $\mathrm{p} 22^{\text {phox }}$ and p47phox, $\mathrm{p}-\mathrm{p} 38 \mathrm{MAPK}$ and $\mathrm{p}-\mathrm{NF}-\mathrm{\kappa B}$, significantly more in the permeable than in the non-permeable group. The samples were derived from the same experiment and gels/blots were processed in parallel. Bar graphs represent data in mean $\pm \mathrm{SD}$ based on 5 experiments, $* \mathrm{P}<0.05$ vs. non-permeable group.

apoptotic cells when the stress is modest (equivalent to 10 to $40 \mu \mathrm{g} /$ $\mathrm{ml}$ concentrations of ox-LDL). At higher concentrations (60 to $100 \mu \mathrm{g} / \mathrm{ml}$ ), ox-LDL leads to significantly increase in non-viable cells and high apoptosis rate that is beyond the protective ability of autophagy-mediated survival mechanism. Actually in these high concentrations of ox-LDL, autophagy itself could serve to enhance cell death $^{7,14}$

As Bcl-2 family of proteins, Bcl-2 and Bcl-xL are well-known antiapoptotic mediators, while Bax and Bak promote apoptosis. It is thought that a ratio of anti-apoptotic and pro-apoptotic members of the Bcl-2 family determines the apoptotic potential of cells ${ }^{21}$. We observed a pronounced expression of pro-apoptotic Bax and diminished expression of anti-apoptotic Bcl-2 and Bcl-xL in HUVECs in the permeable group. The resultant extensive apoptotic activity was confirmed by analysis of Caspases expression. These observations taken together can be interpreted to imply that ox-LDL concentration polarization damages cell surface HSPG, which provides prolonged interaction between ox-LDL and extensive expression of cell surface receptors leading to intense LOX-1 expression followed by ox-LDL uptake in endothelial cells and activation of cell death (apoptosis) and survival (autophagy) pathways. This chain of events is largely, but not entirely, dependent on the expression of LOX-1, since LOX-1 antibody blocks many of the steps postulated in this pathway.

For water filtration rate $\left(v_{w}\right)$ measurement, $v_{w}$ would depend upon the degree of endothelial cell apoptosis. High degree of apoptosis will cause cell death and cell loss, thus result in increased $v_{w}$. Though we noted an increase in apoptosis with increasing concentration of
ox-LDL (20 to $60 \mu \mathrm{g} / \mathrm{ml}$ ), these concentrations only induced moderate apoptosis of endothelial cells without much cell loss. Therefore, the average value of $v_{w}$ remained similar in the permeable group and thoracic aorta.

Experiments with rabbit thoracic aorta showed that concentration polarization of ox-LDL also occurs on the luminal surface, and this is followed by activation of a series signaling pathways akin to those seen in isolated cultured endothelial cells. Although the measurement of $c_{w a}$ instead of $c_{w}$, may not be very accurate, we believe that the trend of ox-LDL accumulation in the arterial wall would be similar to that observed in vivo.

Hemodynamic parameters, particularly associated with low shear stress, oscillatory flow and turbulent flow, play important roles in the localization of atherosclerotic lesions within arteries ${ }^{25}$. Previous studies have shown that blood flow in the aortic arch exhibits a pattern of swirling flow, which may exert some protective effect against formation of atherosclerotic plaques in the $\operatorname{arch}^{26}$. Our study showed that, compared with normal flow, swirling flow protected cell surface HSPG from damage by ox-LDL; Aortic segments with swirling flow also exhibited limited LOX-1 expression, as well as autophagy and apoptosis. It is probable that swirling blood flow washes off atherogenic molecules in the blood so that noxious molecules (such as, oxLDL) cannot accumulate in the wall of the arteries. This may be the basis for relatively infrequent formation of atherosclerotic lesions in the arch and the ascending aorta.

Besides LOX-1 related downstream activities, such as induction of apoptosis and autophagy, we confirmed previously defined upstream 
signals that lead to LOX-1 expression; these include ROS generation, expression of NADPH oxidases (p22 ${ }^{\text {phox }}$ and $\mathrm{p} 47^{\text {phox }}$ subunits), and activation of $\mathrm{p} 38 \mathrm{MAPK}$ and NF- $\mathrm{\kappa B}$. Hence, it can be concluded that concentration polarization of ox-LDL activates these upstream signals as well. This information explains as to why atherosclerosis occurs preferentially in curved areas and in bifurcations which are prone to accumulate large concentrations of atherogenic lipids ${ }^{1,6}$.

In conclusion, the present study provides new insight into our understanding of the role that concentration polarization of oxLDL exerts on HSPG degradation and regulation of autophagy and apoptosis. Clearly, these effects are largely, if not completely mediated by LOX-1 expression. Further, this study provides evidence for the concept that swirling flow in the arterial system has a beneficial effect on the transport of ox-LDL onto the luminal surface of the artery, thereby reducing concentration polarization of ox-LDL and suppressing LOX-1 expression, autophagy and apoptosis.

\section{Methods}

HUVECs monolayer culture experiments. Human umbilical vein endothelial cells (HUVECs) were isolated from human umbilical cords with vascular cell basal medium supplemented with HUVECs Growth Kit (ATCC, Manassas, VA). HUVECs at a density of $1 \times 10^{6}$ cells $/ \mathrm{cm}^{2}$ were seeded on:

i) Non-permeable group: a glass slide which was non-permeable to plasma ii) Permeable group: a Millicell-CM membrane (PICM 03050; Millipore Corp., Bedford, MA)

which was permeable to plasma with pores of $0.4 \mu \mathrm{m}$ in diameter. The state of attachment of cells onto the membrane and confluence of cells on the membranes were monitored by a phase contrast microscope from time to time.

Perfusion system setup. Experimental perfusion system as described previously by Ding et $\mathrm{al}^{19}$ was used (Figure 1A). It consisted of a head tank, a downstream collecting reservoir, a modified parallel-plate flow chamber with a height of $0.5 \times 10^{-3} \mathrm{~m}$, a peristaltic flow pump to circulate the perfusion fluid (cell culture medium) and a humidified atmosphere with $5 \% \mathrm{CO}_{2}$. A pressure transducer and a flow meter were used to monitor the perfusion pressure and the flow rate through the flow chamber, respectively. During the experiment, the flow chamber was kept at a constant temperature of $37^{\circ} \mathrm{C}$.

Wall shear stress was calculated using the formula: $\tau=6 \mu \mathrm{Q} / w h^{2}$, where $\mu$ is the viscosity of the medium and $Q$ is the flow rate, $h$ and $w$ are the width and height of the parallel-plate flow chamber.

During the measurements, steady wall shear stress and perfusion pressure in the flow chamber were kept at $1.3 \mathrm{~Pa}$ and $100 \mathrm{mmHg}$, respectively, while the ox-LDL concentration in the perfusate was varied between 0 to $60 \mu \mathrm{g} / \mathrm{ml}$. The flow was maintained for $24 \mathrm{~h}$ except for Dil-ox-LDL uptake when the flow was for $2 \mathrm{~h}$.

Measurement of filtration rate and DiI-ox-LDL wall concentration of HUVECs. For each experiment, the filtration rate across the wall of cell monolayer on the Millicell-CM membrane was measured as described previously ${ }^{6}$ with the help of the calibrated pipette with inner diameter of $1 \mathrm{~mm}$ supported on the dish (Figure 1A). The concentration of DiI-ox-LDL (Yiyuan Biotech, GZ, China) on the luminal surface of HUVECs layer was assessed by measuring the fluorescence intensity of DiI-ox-LDL with a Confocal Laser Scanning Microscopy (SPII, Leica, Heidelberg, Germany).

Dil-ox-LDL uptake. Cultured endothelial cells were perfused with Dil-ox-LDL $5 \mu \mathrm{g} /$ $\mathrm{ml}$ for $2 \mathrm{~h}$ at $37^{\circ} \mathrm{C}$. Then, the cells were gently washed with PBS three times and digested with Trypsin-EDTA. Dil-ox-LDL uptake was measured using flow cytometry (Becton Dickinson, Franklin Lakes, NJ).

Western blot. Primary and secondary antibodies were purchased from Abcam (San Francisco, CA), Santa Cruz Biotechnology (Santa Cruz, CA) and Novus Biologicals (Littleton, CO). All gels were run and transferred to membrane under same experimental conditions. Details of western blotting have been published earlier ${ }^{9}$.

Measurement of intracellular reactive oxygen species. Intracellular ROS was measured with the use of the fluorescent signal dihydroethidium (DHE), a cell-permeable indicator for ROS generation. Essentially, after $24 \mathrm{~h}$ perfusion, cells were treated with $10 \mu \mathrm{mol} / \mathrm{L}$ DHE (Invitrogen, Grand Island, NY) in PBS for $30 \mathrm{~min}$. The ROS-mediated fluorescence was measured by flow cytometry (Becton Dickinson, Franklin Lakes, NJ), and the results were analyzed with the software WinMDI29.

Experiments with rabbit thoracic aorta. Male New Zealand white rabbits weighing approximately $3.5 \mathrm{~kg}$ were obtained from the Laboratory Animal Center, Peking University (Beijing, China). The experiments followed a protocol approved by the institutional committee on animal use, and all animal care complied with the 'Principles of Laboratory Animal Care' and the 'Guide for the Care and Use of
Laboratory Animals' (NIH Publication No. 86-23, revised 1985). Rabbits were anesthetized intravenously through the right marginal ear vein with a mixture of xylazine $(2.2 \mathrm{mg} / \mathrm{kg})$ and ketamine $(22 \mathrm{mg} / \mathrm{kg})$. Under anesthesia, straight segment of the rabbit thoracic aorta was removed. The arterial segment was bathed in Krebs solution to prevent it from drying.

Measurement of filtration rate and DiI-ox-LDL wall concentration of rabbit thoracic aortas. For all experiments with rabbit thoracic aorta, perfusion pressure and flow rate were kept at $90 \mathrm{mmHg}$ and $40 \mathrm{ml} / \mathrm{min}$, respectively; both represent physiologic values in the rabbit thoracic aorta. The filtration rate across the wall of thoracic aorta was measured as described by Deng et $\mathrm{al}^{6}$.

Due to the difficulty in measuring the wall (luminal surface) concentration of DiIox-LDL $\left(c_{w}\right)$ in the thoracic aortic segment, instead of $c_{w}$, we measured DiI-ox-LDI surface absorption concentration $\left(c_{w a}\right)$ assuming that $c_{w a}$ approximates with $c_{w}$. After perfusion, thoracic aortic segment was flushed with phosphate-buffered saline (PBS) buffer to wash off the dissociative DiI-ox-LDL, and fixed with a modified Karnovsky's fixative $(2.5 \%$ glutaraldehyde and $2 \%$ paraformaldehyde in $0.15 \mathrm{M}$ sodium cacodylate buffer, $\mathrm{pH} \mathrm{7.4)}$ ) at $90 \mathrm{mmHg}$ for $6 \mathrm{~h}$ in the dark. Then $c_{w a}$ was assessed by Confocal Laser Scanning Microscopy (SPII, Leica, Heidelberg, Germany).

Analysis of apoptosis in endothelial cells and rabbit thoracic aortas. Apoptosis was analyzed by Western blotting and Polycaspase FLICA apoptosis detection kit (ImmunoChemistry Technologies, Bloomington, MN) according to supplied protocols. Total caspase activity was assessed by flow cytometry (Becton Dickinson, Franklin Lakes, NJ), and the results were analyzed with WinMDI29 software (Becton Dickinson).

Autophagy detection in endothelial cells and rabbit thoracic aortas. Autophagy was detected by Western blot, Premo ${ }^{\mathrm{TM}}$ Autophagy Sensors (L3B-GFP) *BacMam $2.0 *$ (Invitrogen, Grand Island, NY) by fluorescence microscopy and flow cytometry according to supplied protocols.

Characterization of flow in the rat thoracic aorta. To study the effect of swirling flow on ox-LDL concentration polarization in the wall of the thoracic aorta, flow generated in the test vessel with the spiral flow guider was characterized numerically and compared with the number in the normal flow model. The model for the spiral flow study was a rigid vessel combined with the spiral flow guider, whereas model for the normal flow study was a rigid vessel without the flow guider. The length and the inner diameter of both vessels were $20 \mathrm{~mm}$ and $3 \mathrm{~mm}$, respectively. The perfusion solution in the present study was cell culture medium, which was assumed to be homogeneous, incompressible and Newtonian fluid. Its viscosity and density were 1.3 $\times 10^{-3}$ and $1047 \mathrm{~kg} / \mathrm{m}^{3}$, respectively. For flow simulation, the Reynolds number was set at 650 . The boundary conditions were set as follows: (1) because flow disturbance occurs at the inlet of the models due to the connection, a uniform velocity profile was assumed at the inlet based on the flow rate of $40 \mathrm{~mL} / \mathrm{min}$ used in the experiment; (2) the outlet condition was set to be outflow, which applied a zero diffusion flux condition like a fully developed flow; and, (3) the wall of the vessels was assumed to be rigid and non-slippery.

The numerical simulation was based on the three dimensional incompressible Navier-Stokes equations as follows:

$$
\begin{gathered}
\rho(\vec{u} \cdot \nabla) \vec{u}+\nabla p-\mu \Delta \vec{u}=0 \\
\nabla \cdot \vec{u}=0
\end{gathered}
$$

where $\vec{u}$ is the fluid velocity vector, $\rho$ and $\mu$ are the density and viscosity of the perfusion solution, and $p$ is the pressure.

Finite volume method was used in the simulation. The computational meshes of the models created using the CAD software Gambit 2.2 (ANSYS, Inc., Canonsburg, PA) were all unstructured hexahedron grid. The commercially available computational fluid dynamics (CFD) code, FLUENT 6.2 (ANSYS, Inc.) was employed in the numerical simulation. Discretization of the pressure and momentum at each control volume was in a second-order scheme. The iterative process of computation was terminated when the residual of mass and velocity were all less than the convergence criterion, $1.0 \times 10^{-5}$

Statistical analysis. Statistical analysis was performed with SPSS 11.5 software. Data are presented as means and standard deviation (SD) from 5-7 independent experiments. Univariate comparisons of means were evaluated using the Student $t$ test, $\mathrm{P}<0.05$ was considered statistically significant.

1. Nigro, P., Abe, J. \& Berk, B. C. Flow shear stress and atherosclerosis: a matter of site specificity. Antioxid Redox Signal 15, 1405-1414 (2011).

2. Davies, P. F. Flow-Mediated Endothelial Mechanotransduction. Physiol Rev $\mathbf{7 5}$ 519-560 (1995)

3. Vincent, P. E., Sherwin, S. J. \& Weinberg, P. D. The effect of a spatially heterogeneous transmural water flux on concentration polarization of low density lipoprotein in arteries. Biophys J 96, 3102-3115 (2009).

4. Tedgui, A. \& Lever, M. J. Filtration through damaged and undamaged rabbit thoracic aorta. Am J Physiol 247, H784-791 (1984). 
5. Bratzler, R. L., Chisolm, G. M., Colton, C. K., Smith, K. A. \& Lees, R. S. The distribution of labeled low-density lipoproteins across the rabbit thoracic aorta in vivo. Atherosclerosis 28, 289-307 (1977).

6. Deng, X. et al. Luminal surface concentration of lipoprotein (LDL) and its effect on the wall uptake of cholesterol by canine carotid arteries. J Vasc Surg 21, 135-145 (1995).

7. Kataoka, H. et al. Expression of lectinlike oxidized low-density lipoprotein receptor-1 in human atherosclerotic lesions. Circulation 99, 3110-3117 (1999).

8. Sawamura, T. et al. An endothelial receptor for oxidized low-density lipoprotein. Nature 386, 73-77 (1997).

9. Chen, J. W., Mehta, J. L., Haider, N., Zhang, X. J., Narula, J. \& Li, D. Role of caspases in ox-LDL-induced apoptotic cascade in human coronary artery endothelial cells. Circ Res 94, 370-376 (2004).

10. Lu, J., Mitra, S., Wang, X., Khaidakov, M. \& Mehta, J. L. Oxidative stress and lectin-like ox-LDL-receptor LOX-1 in atherogenesis and tumorigenesis. Antioxid Redox Signal 15, 2301-2333 (2011)

11. Levine, B., Mizushima, N. \& Virgin, H. W. Autophagy in immunity and inflammation. Nature 469, 323-335 (2011).

12. Mizushima, N., Levine, B., Cuervo, A. M. \& Klionsky, D. J. Autophagy fights disease through cellular self-digestion. Nature 451, 1069-1075 (2008).

13. Green, D. R., Galluzzi, L. \& Kroemer, G. Mitochondria and the autophagyinflammation-cell death axis in organismal aging. Science 333, 1109-1112 (2011).

14. Ding, Z., Liu, S., Wang, X., Khaidakov, M., Dai, Y. \& Mehta, J. L. Oxidant stress in mitochondrial DNA damage, autophagy and inflammation in atherosclerosis. Sci Rep 3, 1077 (2013).

15. Ding, Z et al. Regulation of autophagy and apoptosis in response to ox-LDL in vascular smooth muscle cells, and the modulatory effects of the microRNA hsa-let-7g. Int J Cardiol (2013). doi:pii: S0167-5273(12)01674-9. 10.1016/ j.ijcard.2012.12.045. [Epub ahead of print].

16. Conforti, G. et al. Human endothelial cells express integrin receptors on the luminal aspect of their membrane. Blood 15, 437-446 (1992).

17. Resnick, N. \& Gimbrone, M. A. Jr. Hemodynamic forces are complex regulators of endothelial gene expression. FASEB J 9, 874-882 (1995).

18. Chiu, J. J. \& Chien, S. Effects of disturbed flow on vascular endothelium: pathophysiological basis and clinical perspectives. Physiol Rev 91, 327-387 (2011)

19. Ding, Z., Liu, S., Yang, B., Fan, Y. \& Deng, X. Effect of oxidized low-density lipoprotein concentration polarization on human smooth muscle cells' proliferation, cycle, apoptosis and oxidized low-density lipoprotein uptake. JR Soc Interface 9, 1233-1240 (2012).

20. Häcker, U., Nybakken, K. \& Perrimon, N. Heparan sulphate proteoglycans: the sweet side of development. Nat Rev Mol Cell Biol 6, 530-541 (2005).
21. Elmore, S. Apoptosis: a review of programmed cell death. Toxicol Pathol 35, 495-516 (2007)

22. Morbiducci, U., Ponzini, R., Grigioni, M. \& Redaelli, A. Helical flow as fluid dynamic signature for atherogenesis risk in aortocoronary bypass. A numeric study. J Biomech 40, 519-534 (2007).

23. Davies, P. F. Hemodynamic shear stress and the endothelium in cardiovascular pathophysiology. Nat Clin Pract Cardiovasc Med 6, 16-26 (2009).

24. Oka, T. et al. Mitochondrial DNA that escapes from autophagy causes inflammation and heart failure. Nature 485, 251-255 (2012).

25. Malek, A. M., Alper, S. L. \& Izumo, S. Hemodynamic shear stress and its role in atherosclerosis. JAMA 282, 2035-2042 (1999).

26. Heil, M. \& Schaper, W. Influence of mechanical, cellular, and molecular factors on collateral artery growth (arteriogenesis). Circ Res 95, 449-458 (2004).

\section{Acknowledgments}

We are grateful to $\mathrm{Dr}$ Qinghua $\mathrm{Hu}, \mathrm{PhD}$ (Beihang University) for her comments and advice on several aspects of the experimental work. This work is supported by Grants-in-Aid from the National Natural Science Foundation of China (No. 31170904, 11228205 and 61190123), Specialized Research Fund for the Doctoral Program of Higher Education of China (20121102110031) and the Department of Veterans Affairs, Washington, DC

\section{Author contributions}

Z.D. and S.L. developed the concept of this study and performed the experiments; C.S., Z.S. and Y.F. performed immunohistochemical analyses; X.W. helped with autophagy experiments; X.D. and J.L.M. designed the study, analyzed the data and wrote the manuscript. All authors read and approved the final manuscript.

\section{Additional information}

Competing financial interests: The authors declare no competing financial interests. How to cite this article: Ding, Z. et al. Concentration polarization of ox-LDL activates autophagy and apoptosis via regulating LOX-1expression. Sci. Rep. 3, 2091; DOI:10.1038/ srep02091 (2013)

(i) This work is licensed under a Creative Commons Attributioncc. visit http://creativecommons.org/licenses/by-nc-nd/3.0 